\title{
Development and Testing of the Psychometric Properties of the Attitude Towards Medical Device-related Pressure Ulcers/Injuries Questionnaire Thila Fereidounia, Mohammad Behnammoghadam ${ }^{b}$, Farnoosh Rashvand ${ }^{c}$, Hossein Rafiei ${ }^{c}$ ${ }^{\text {a}}$ Fasa University of Medical Sciences, Fasa, Fars, Iran \\ b Yasuj University of Medical Sciences, Yasuj, Iran \\ ${ }^{c}$ Qazvin University of Medical Sciences, Qazvin, Iran
}

Abstract

BACKGROUND: Medical device-related pressure ulcers/injuries (MDRPU/Is) are a serious concern in health care. PURPOSE: To develop and assess the psychometric testing of a questionnaire to measure nursing students' attitudes about the care and prevention of MDRPU/Is. METHODS: Based on a review of the literature, a 26-item questionnaire was developed; face validity was assessed by ten (10) nursing students. The modified Lawshe's model was used, and both the content validity index (CVI) and content validity ratio were calculated. Items with a CVI more than 0.63 were retained. The minimum optimal CVI for the new instrument was $79 \%$. Qualitative assessments were performed by 10 experienced faculty members. One-hundred-andeighty seven (187) nursing students participated in the construct validity testing of the 11-item questionnaire. The Kaiser-Meyer-Olkin test of sampling adequacy and Bartlett's test of sphericity were performed. Following analysis of the main components and the varimax rotation, the factor analysis was determined. Internal consistency (Cronbach's alpha) and test-retest were determined using Pearson's correlation and intraclass correlation coefficient to evaluate reliability. RESULTS: During the face validity phase of the initial 26 items, 7 items had impact scores less than 1.5. After calculating the CVI and content validity ratio for all items, 8 items did not achieve the desirable score. After performing exploratory factor analysis on the remaining 11 items, the Kaiser-MeyerOlkin test value was 0.789, and Bartlett's test of sphericity was 0.0001, which was statistically significant. Internal consistency of items (Cronbach's alpha of 0.77) showed that all items had a high correlation. The reliability of test-retest was significant using an intraclass correlation coefficient of 0.75 and Pearson's correlation coefficient of 0.86 at $\mathrm{P}<.005$. CONCLUSION: In this sample of nursing students, the Attitude Towards Medical-device Related Pressure Ulcers/Injuries Questionnaire was valid and reliable. Studies including licensed clinicians are needed to confirm these results. 